\title{
A Novel Touch Probe Triggered by Harmonic Generation
}

\author{
TAKAHASHI Satoshi ${ }^{1}$, MIZUMOTO Hiroshi ${ }^{1}$, ARII Shiroh ${ }^{1}$, YABUYA Makoto ${ }^{2}$ \\ ${ }^{1}$ Tottori University, Koyama, Tottori, 680-8552, Japan \\ ${ }^{2}$ Nachi-Fujikoshi Corp., Namerikawa, Toyama, 936-0802, Japan
}

\begin{abstract}
:
A new type of touch probe for measuring small optical parts is proposed. The probe is excited by a piezoelectric actuator in sinusoidal waveform. When the probe touches an object, harmonic components of the excitation appear in the power spectrum of the probe vibration. It is judged that the probe touches the object at the power of the second harmonic exceeds a threshold level. The touch probe is mounted on a small CMM and evaluated its performance by measuring standard steel balls.
\end{abstract}

Key words: CMM, FFT, On-machine measurement, Piezoelectric actuator, Touch probe

\section{Introduction}

The demand for the three dimensional (3D) measurement and evaluation of small objects made by ultraprecision machine tools is ever increasing. Ideally, these small objects, the micro pickup lens for DVD and its die for examples, should be measured on the machine tool machining the objects [1]. In the present paper, we propose a new type of touch probe for the on-machine measurement of small objects. Touch probes used in the conventional coordinate measuring machine (CMM) can be divided into two types [2]. The first type uses a kind of an electric switching circuit that is cut by the mechanical force generated as the tip of the probe pushes the object. This type of the probe is simple in structure, however, considerable measuring force is needed for stable measurement. High measuring force will be harmful for the optical surface of the lens die. The second type uses a harmonic vibration excited by a piezoelectric actuator or an induction coil. When the tip of the vibrating probe touches an object, there occurs some change in the vibration mode. Conventional vibrating touch probe detects the decrease in the vibration amplitude when the touching occurs. The measuring force at touching can be less than the first switching type, however, measuring resolution is not high enough.

The vibrating touch probe proposed in the present paper utilizes the harmonic component of the excited vibration. When the touch probe approaches the object, the second harmonic appears immediately after the touching. The measuring force can be less than that of conventional touch probes. Therefore, the proposed touch probe can measure the object with less damage, and higher measuring performance is expected.

\section{Principle of Touch Detection}

Figure 1 (a) shows a sinusoidal wave (upper) and its power spectrum (lower), where only one line is seen at the fundamental frequency $f_{1}$ of the sinusoidal wave. When the top of the sinusoidal wave is flattened as shown in Fig.1 (b) upper, harmonics $f_{n}(n=2,3,0 \mathrm{~s})$ can be seen in the power spectrum. Namely, if some distortion occurs in the sinusoidal waveform, the harmonics appears in its spectrum.

The touch probe proposed in the present paper utilizes this change in the spectrum to detect the touching of the probe to an object. When the probe contacts the object, the waveform of the oscillation is distorted slightly as shown in Fig.1 (b). Therefore, by monitoring the appearance of the harmonics in the power spectrum of the vibration, one can easily know the probe touch to the object. We decide that the probe touches the object when the power of the second harmonic $f_{2}$ exceeds a threshold level. This criterion triggered by harmonic generation is effective to detect the touching with less measuring force than that of conventional touch probes. The power spectrum of the probe vibration can be calculated in real-time by using FFT algorithm.

\section{Experimental Method}

\subsection{Experimental Apparatus}

The block diagram of a 3D measuring system using the proposed vibrating touch probe triggered by the harmonic generation is shown in Fig.2, and the photo of a small CMM with the touch probe is shown in Fig.3. Figure 4 shows a close-up view of the touch probe and a standard steel ball to be measured for the evaluation of the performance of the touch probe.

A steel probe having a ceramic ball tip of $0.6 \mathrm{~mm}$ in diameter is attached at the end of a steel cantilever. A piezoelectric actuator (PZT 1) fixed on the backside of the cantilever is used as a vibrator. Maximum elongation of the piezoelectric actuator is about $4 \mu \mathrm{m}$ at the maximum applied voltage of $150 \mathrm{~V}$, and the resonant frequency of the actuator is $260 \mathrm{kHz}$. For the sinusoidal excitation of the probe, a function generator shown in Fig.2 below is used. The sinusoidal signal generated is input to the PZT 1 via the amplifier of the piezoelectric actuator. Gain of the amplifier is fifteen times. The 

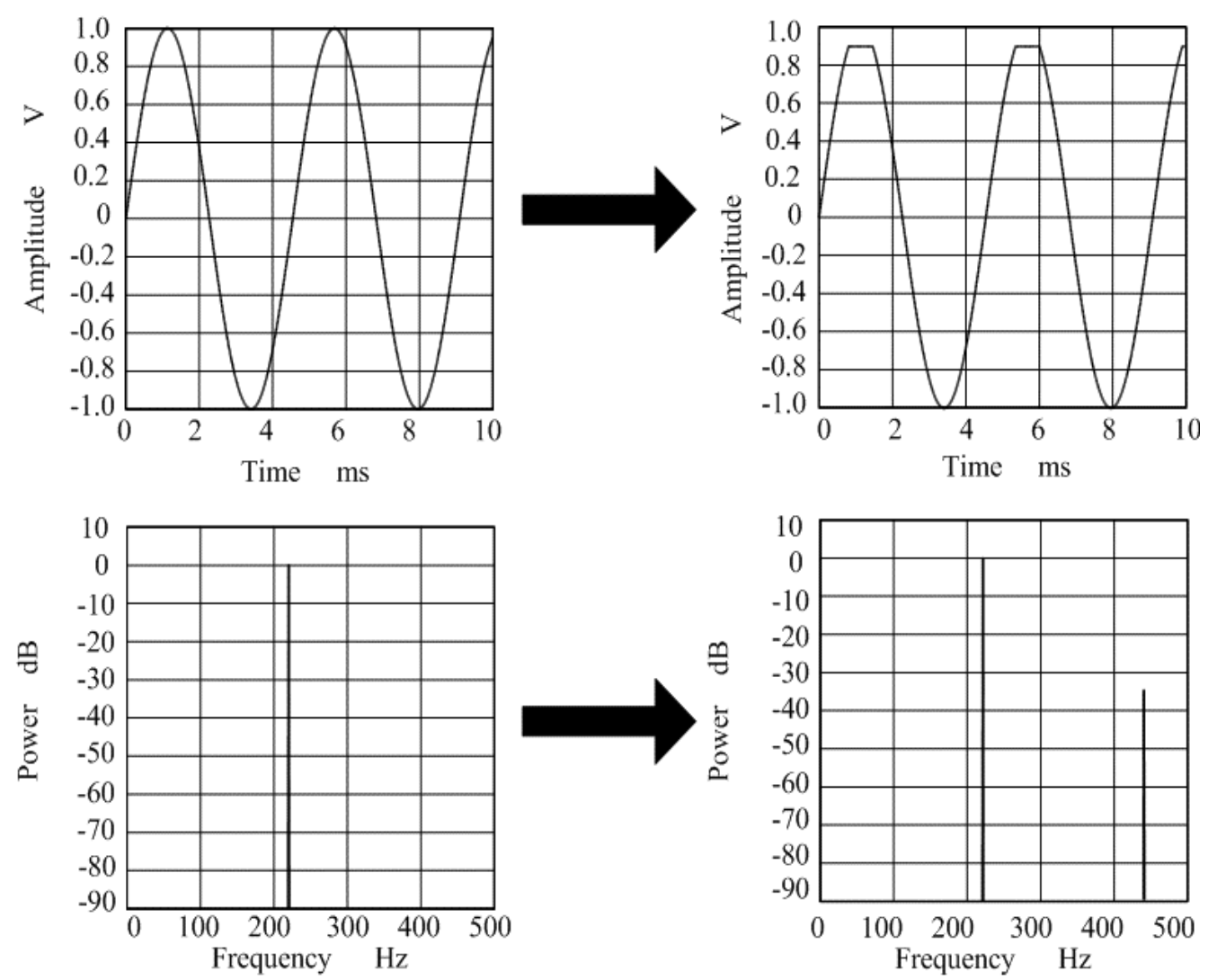

Fig. 1 Distortion of sinusoidal vibration and appearance of second harmonic $\left(f_{1}=220 \mathrm{~Hz}, \quad f_{2}=440 \mathrm{~Hz}\right)$

sinusoidal vibration of the probe induced by the PZT 1 is measured by a capacitance displacement sensor shown (Sensor 1). Measuring range and resolution of the capacitance sensor are $50 \mu \mathrm{m}$ and $10 \mathrm{~nm}$, respectively. Measured signal of the probe vibration is fed to a microcomputer (PC) via an $\mathrm{AD}$ converter; resolution of the $\mathrm{AD}$ converter is 16 bit and the conversion cycle time is $30 \mu \mathrm{s}$. The probe is mounted on a micro-stage driven by a piezoelectric actuator (PZT 2). The stage is supported by leaf springs and the movement of the micro-stage is measured by another capacitance displacement sensor (Sensor 2). The stroke of this micro-stage is about $40 \mu \mathrm{m}$. The micro-stage is mounted on the horizontal Z stage of a XYZ-table, and this $Z$ stage is mounted on the horizontal X stage. An object to be measured is mounted on the vertical $Y$ stage. Each stage is driven by a servomotor controlled by a motor controller connected to the PC.

The PC controls the automated probe driving and contact detecting procedure. At the beginning of this procedure, the vibrating probe is placed apart from the object by driving the $\mathrm{X}, \mathrm{Y}$ and $\mathrm{Z}$ stages. From this starting point, the PC moves the micro-stage mounting the probe in Z-direction toward the object; the movement of the micro-stage is controlled by PZT 2 driven by the analog voltage output from the PC via a DA converter. The position of the micro-stage is measured by the Sensor 2. Measured stage position is input to the PC

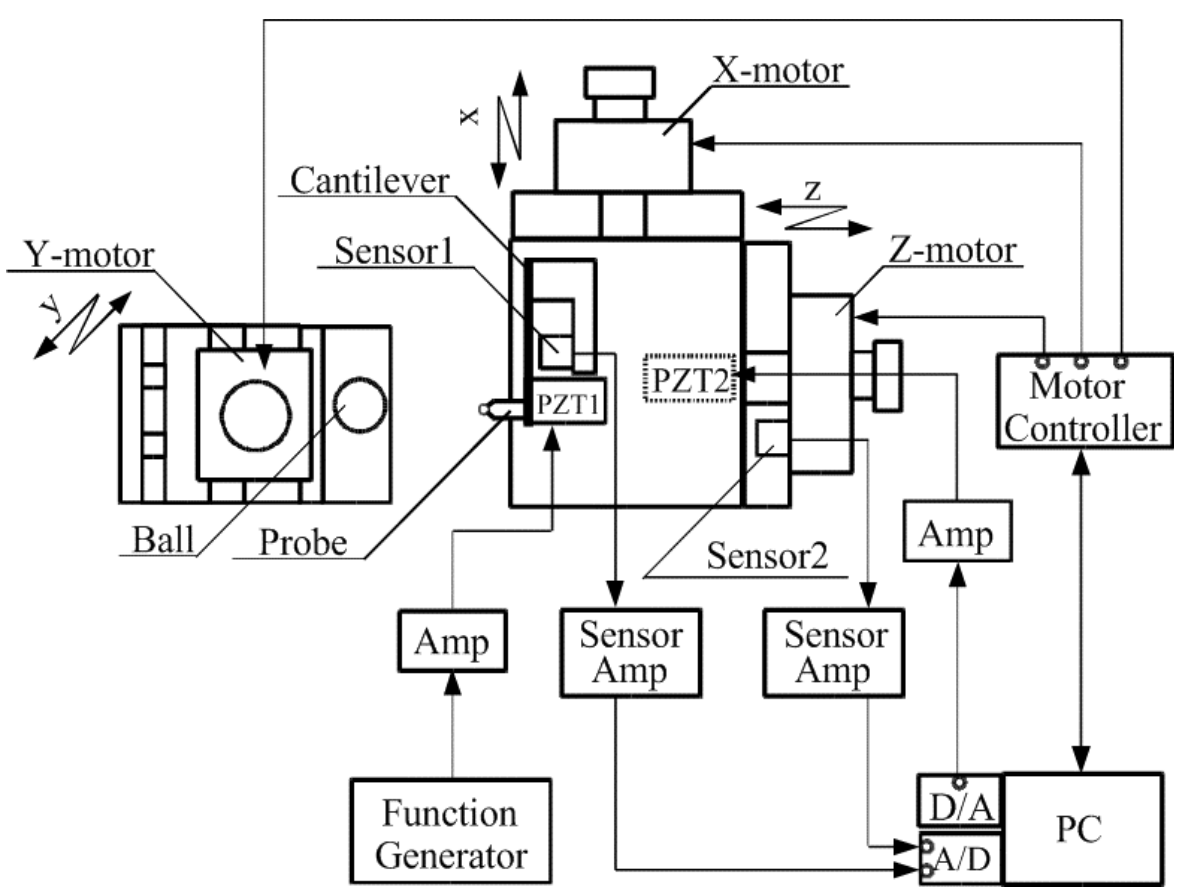

Fig. 2 Measuring system of vibrating touch-probe utilizing second harmonic of excitation 
via the $\mathrm{AD}$ converter. By using FFT algorithm, the PC analyzes the frequency components of the probe vibration, and while the power of the second harmonic of the probe vibration is less than a threshold level, the PC continues to move the micro-stage. When the power of the second harmonic exceeds the threshold level, the PC stops the micro-stage and records the current position of the micro-stage as the contact point of the touch probe. Then the probe is retracted to the starting point by using the micro-stage, and then the probe is moved to the next position to be measured by driving the $\mathrm{X}, \mathrm{Y}$ and $\mathrm{Z}$ stages.

\subsection{Measurement of Small Balls}

At the first step of the measurement, the tip of the probe is aligned to the coordinate of equator of a standard steel ball to be measured by using the horizontal $\mathrm{X}$ stage and the vertical $\mathrm{Y}$ stage. To start the automated probe driving and contact detecting procedure explained in Sec.3.1, the tip is moved carefully toward the ball by using the $\mathrm{Z}$ stage until the gap between the tip and the surface of the ball becomes to be several micrometers.

Then the function generator outputs a sinusoidal signal of $250 \mathrm{~Hz}$ to the PZT 1 for probe vibration, and the PC starts the automated measuring procedure. Figure 4 (a) shows the power spectrum of the probe vibration before contact. Clear line spectrum can be seen at $250 \mathrm{~Hz}$; this line indicates the fundamental frequency of probe vibration. Therefore, the probe vibrates in almost perfect sinusoidal form. When the probe contacts the steel ball, second harmonic of the vibration $(500 \mathrm{~Hz})$ appears in the power spectrum as shown in Fig.4 (b). The power of the second harmonic increases as the probe advances further to the ball. By considering the sensor noise, the threshold level of the power for detecting the second harmonic is decided to be $-70 \mathrm{~dB}$. The level of the sensor noise is about $-80 \mathrm{~dB}$, and lower threshold level will cause the misjudging of the contact. From the decrement in the amplitude of the probe vibration, the measuring force is calculated to be $50 \mu \mathrm{N}$. Higher threshold level causes higher measuring force. When the power of the second

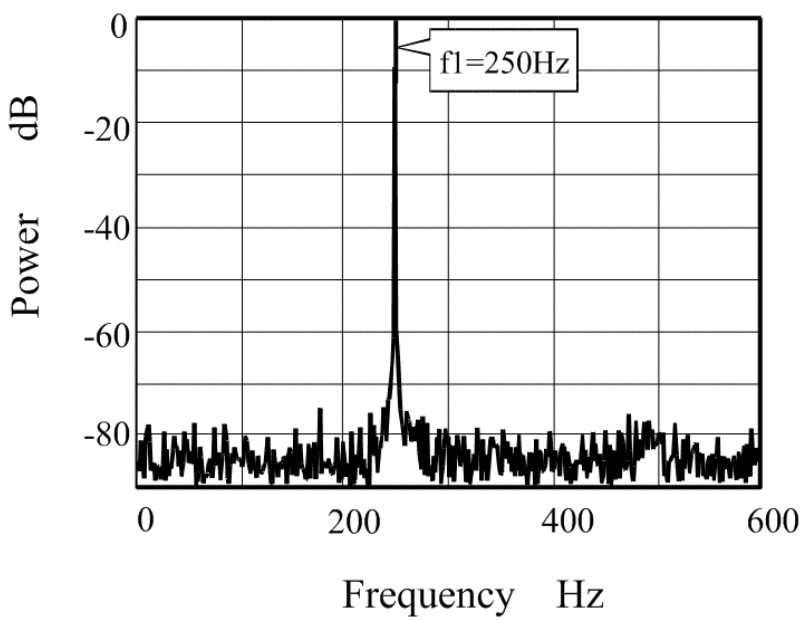

(a)Power spectrum before contact harmonic exceeds $-70 \mathrm{~dB}$, the PC records the position of the probe and ends the automated procedure. For the measurement of next position on the ball, the touch probe is moved by a constant amount in X-direction by using the $\mathrm{X}$ stage. Then, the $\mathrm{Z}$ stage arranges the tip of the probe to the starting point of the next measurement. Such the automated procedure is repeated and the position data of the object shape are stored in the PC.

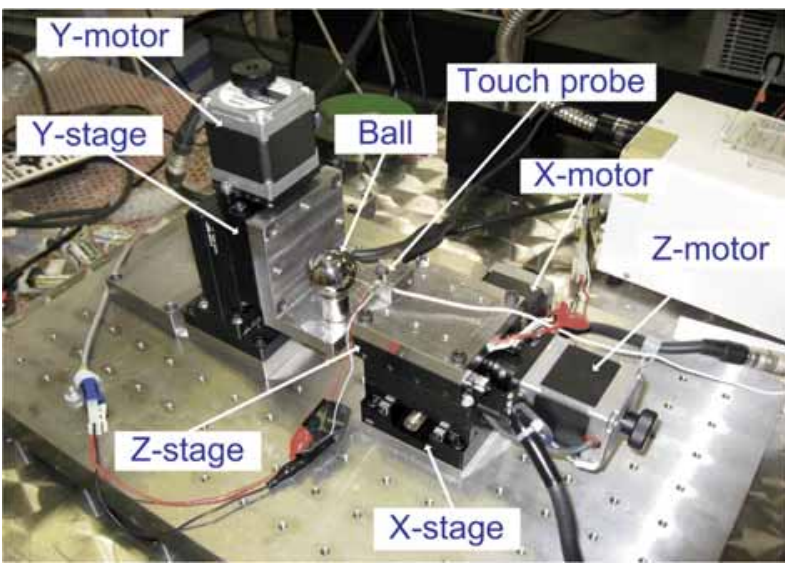

Fig. 3 Small CMM mounting vibrating touch-probe

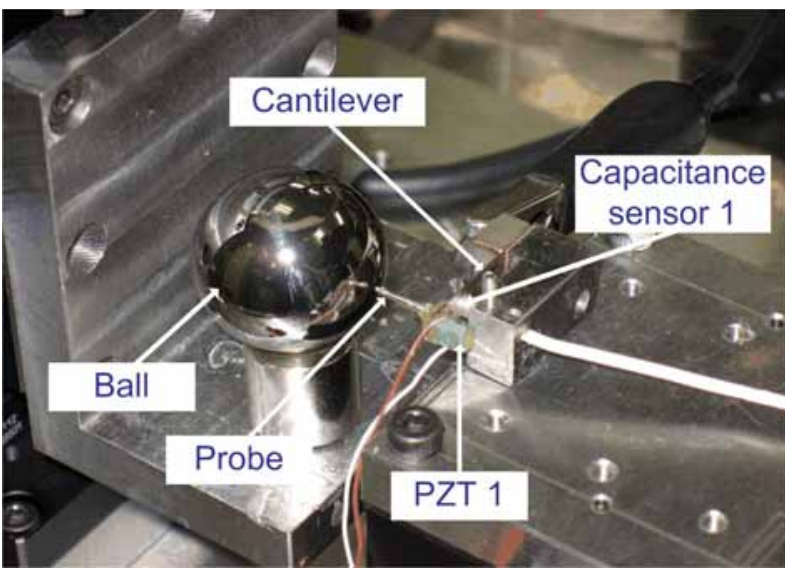

Fig. 4 Vibrating touch-probe and object

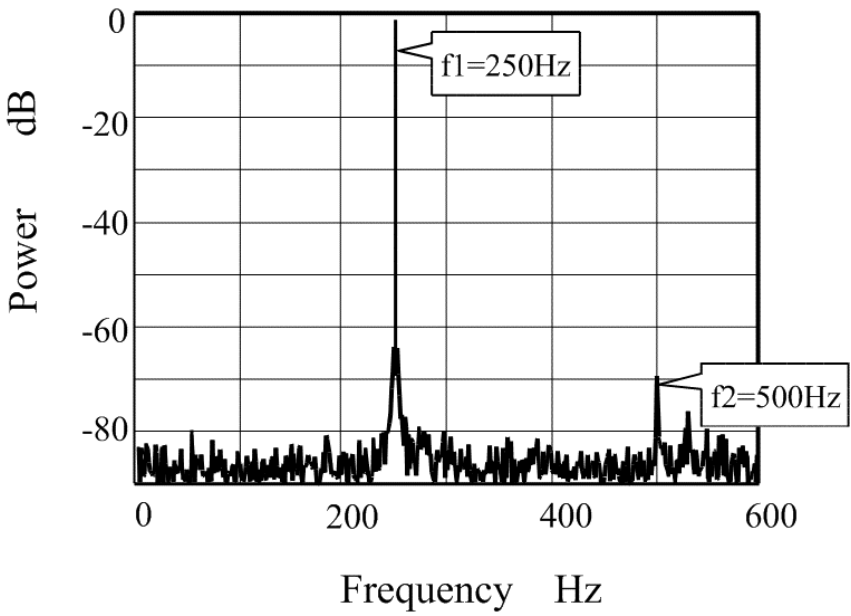

(b)Power spectrum at contact 


\section{Measurement and Discussion}

Instead of small lens dies, standard steel balls of $2 \mathrm{~mm}$ and $25.4 \mathrm{~mm}$ in diameters are measured. The result of measurement for the $25.4 \mathrm{~mm}$-ball is shown in Fig. 6, where the measured positions, the regression curve of the measured points and the theoretical circular arc are illustrated. These position data include the radius of the probe tip $(0.3 \mathrm{~mm})$, therefore, this tip radius should be subtracted from the radius of the regression curve for evaluating the ball radius. Thus evaluated ball radius is $12.66 \mathrm{~mm}$, and the form error is about $0.04 \mathrm{~mm}$. Individual error in each measured value is less than $2 \mu \mathrm{m}$ when the inclination of measured surface, i.e. the contact angle of the probe is small. The error becomes larger when the contact angle increases. Maximum measurement error is $4.8 \mu \mathrm{m}$ at the contact angle of -23degrees.

The result of measurement for the small $2 \mathrm{~mm}$-ball is shown in Fig. 7, where the maximum contact angle is much larger than that of the $25.4 \mathrm{~mm}$-ball. Measured radius of the ball is $0.997 \mathrm{~mm}$ and the form error is about $0.03 \mathrm{~mm}$. Individual error each the measured value is also less than $2 \mu \mathrm{m}$ when the contact angle is small. The error becomes larger when the contact angle increases and maximum measurement error is $4.0 \mu \mathrm{m}$ at the contact angle of -50degrees.

These measurements indicate that the form accuracy of the measuring system is in the range of $0.01 \mathrm{~mm}$ for both balls, and the maximum contact angle of the probe is about 50degrees. The measuring error for individual point is several micrometers, and the error has a week tendency to increase with the contact angle. Therefore, with the small measuring force, the proposed vibrating touch probe is suitable for measuring small optical parts with steep surface.

\section{Conclusion}

A new type of vibrating touch probe is proposed where the appearance of second harmonic triggers the touch detection. Experimental results of measuring small steel balls are summarized as follows:

(1) The shape of the ball can be evaluated with $0.01 \mathrm{~mm}$ order of accuracy.

(2) The measuring error of individual point is several micrometers. Maximum contact angle of the measurement is 50degrees and the error increases slightly with the contact angle.

Therefore, the proposed vibrating touch probe is suitable for the 3D measurement of small parts such as the pick-up lens and its die for DVD.

\section{Acknowledgement}

The authors thank to the Innovation Center for Engineering Education of Tottori University for their kind cooperation in making the experimental apparatus including the vibrating touch probe.

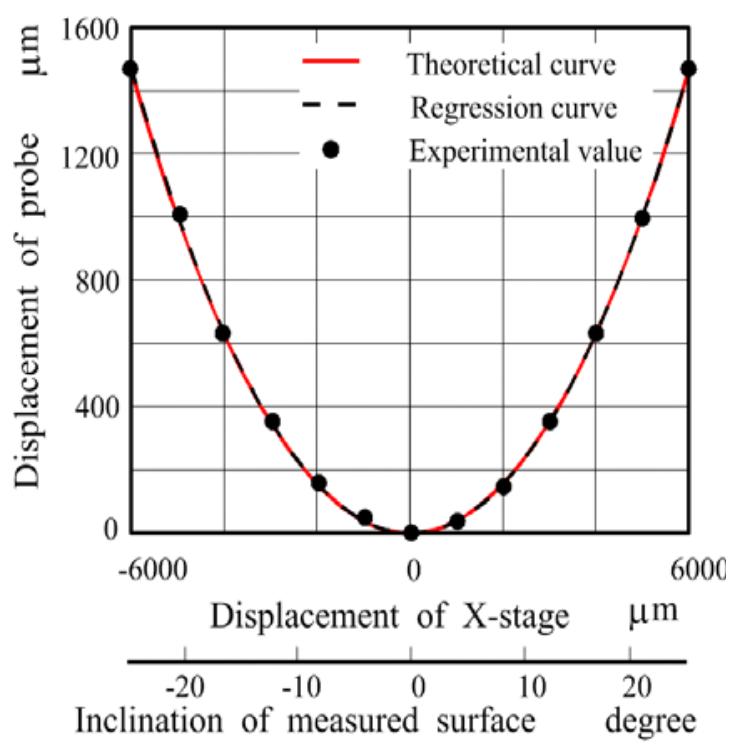

Fig. 6 Profile measurement of $25.4 \mathrm{~mm}$-ball

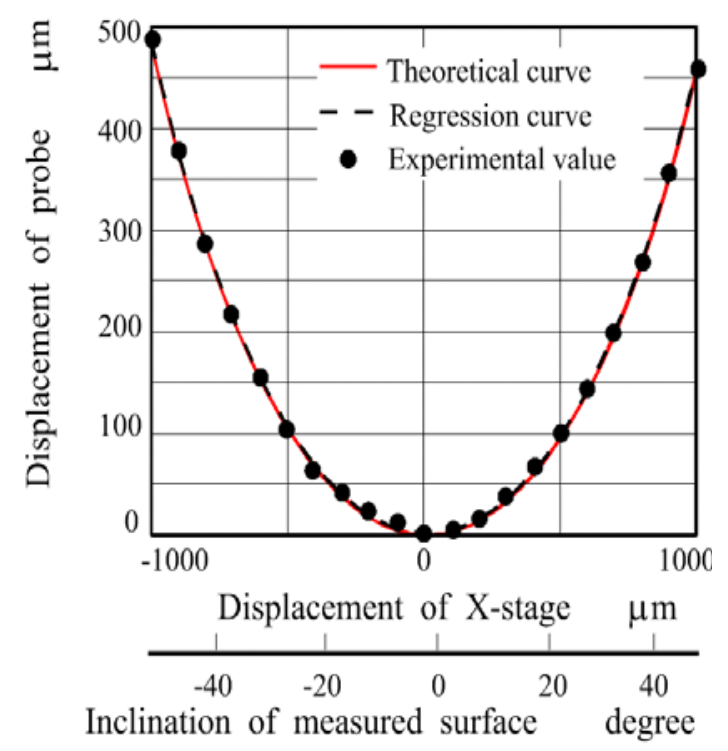

Fig. 7 Profiles measurement of 2mm-ball

\section{References}

[1] Homepage of Nachi-Fujikoshi Corp, http://www.nachi-fujikoshi.co.jp/eng/sei/kakouki/in dex. html.

[2] Dotson, C. et al., Fundamentals of Dimensional Metrology, $4^{\text {th }}$ edition, Thomson (2003) pp.529. 